\title{
Evaluation of the Effects of a New Cosmetic Protocol based on the Application of a Home Peel with $1 \%$ Retinol
}

\author{
Truchuelo $\mathrm{MT}^{1, *}$, Hashtroody $\mathrm{B}^{2}$, and Vitale $\mathrm{M}^{3}$ \\ ${ }^{1}$ Dermatologist MD, PHD in Vithas Nuestra Señora de América Hospital, Madrid, Spain \\ ${ }^{2}$ Scientific and medical affairs director, Medical Department of Cantabria labs, Madrid, Spain \\ ${ }^{3}$ Dermatologist MD and Medical Manager Dermatology at Cantabria labs, Madrid, Spain
}

*Corresponding author: Truchuelo MT, Dermatologist MD, PHD in Vithas Nuestra Señora de América Hospital, Madrid, Spain, Tel: 34606516077; Fax: +34913735088; E-mail: maytetd@yahoo.es

Received: 11 Nov, 2019 | Accepted: 22 Nov, 2019 | Published: 27 Nov, 2019

Citation: Truchuelo MT, Hashtroody B, Vitale M (2019) Evaluation of the Effects of a New Cosmetic Protocol based on the Application of a Home Peel with 1\% Retinol. J Clin Cosmet Dermatol 3(2): dx.doi.org/10.16966/2576-2826.142

Copyright: (C) 2019 Truchuelo M, et al. This is an open-access article distributed under the terms of the Creative Commons Attribution License, which permits unrestricted use, distribution, and reproduction in any medium, provided the original author and source are credited.

\begin{abstract}
Introduction: The main purpose of this study was to determine the safety and tolerance of a new cosmetic procedure based on the use of a Home Peel with $1 \%$ retinol. Secondly, the subjective efficacy was evaluated. The treatment was to be considered well-tolerated if it permitted recovery of Trans Epidermal Water Loss (TEWL) within a few days following the procedure and if patients reported no significant adverse effects. This new protocol was to be judged effective if it improved clinical alterations associated with photoaging.
\end{abstract}

Methods: 6 subjects ( 45 years, Female) with moderate aging were treated with a protocol that included a home peel applied twice daily for 3 days. The protocol included a preparatory and post-procedure period. Pictures to enable clinical evaluation were taken with a Reveal ${ }^{\circledR}$ evice and TEWL was assessed by Tewameter ${ }^{\circledast}$. The subjective evaluation of the subjects and clinical improvement from the investigator was also registered.

Results: A moderate improvement described by both investigator and patients was observed in $83 \%$ of subjects. Tolerance was excellent, with low grade of desquamation, erythema and burning sensation after the preparation phase (TO) and immediately following the home peel (T3).

Conclusion: A new protocol including a home peel product with retinol demonstrated to be safe and effective in the management of skin aging. Improvement in the signs of aging was perceived by both patients and the investigator, with excellent tolerance.

Keywords: Cosmetic; Peeling; Aging; Retinol; Treatment; Rejuvenation

\section{Background}

Home devices are increasingly demanded; they complement dermatological treatments performed in the dermatologist's office and help increasing efficacy by maintaining treatment over a longer period of time. For a home care treatment to be successful, it must be easy to use, effective and well tolerated. It should also have predictable results. The current trend in photoaging treatment is to combine different options so that synergies provide a greater increase in the clinical skin appearance improvement. One of the most effective pharmacological Treatment reported in scientific literature are topical retinoic acid (tretinoin) and with respect to cosmetic treatment, retinol and Alpha Hydroxy Acids (AHA) [1].

Retinoids represent the topical option that has been shown to be most effective in photoaging treatment in clinical trials. Clinical improvement observed in dyschromia, roughness and skin wrinkles results from the activity of retinoids on nucleus receptors and cell cytoplasm [2]. The effectiveness of retinoids in pigmentation has been attributed to an increase in keratinocyte turnover as well as a decrease in melanin transfer [3]. In addition, it favors the penetration of other active substances.

Retinoids act directly by binding to receptors: Retinoic Acid Receptors (RAR) and X Receptors for Retinoids X (RXR). RAR and RXR belong to the super family of nuclear hormone receptors (which also includes steroid receptors, Vitamin D, thyroid hormone, and others). They have alpha, beta and gamma subtypes with different isoforms for each subtype. Synthetic retinoids bind selectively to the retinoid receptor. The binding to these nuclear receptors induces a modification of DNA signaling and expression of cell differentiation and proliferation genes. These changes determine the ability to normalize the activity of melanocytes and thus regulate the depigmentation associated with photoaging, tone evening and reducing dark spots and lentigines [4].

As previously described, Retinoic acid improves skin aging. However, less it is known about the anti-aging effects of retinol 


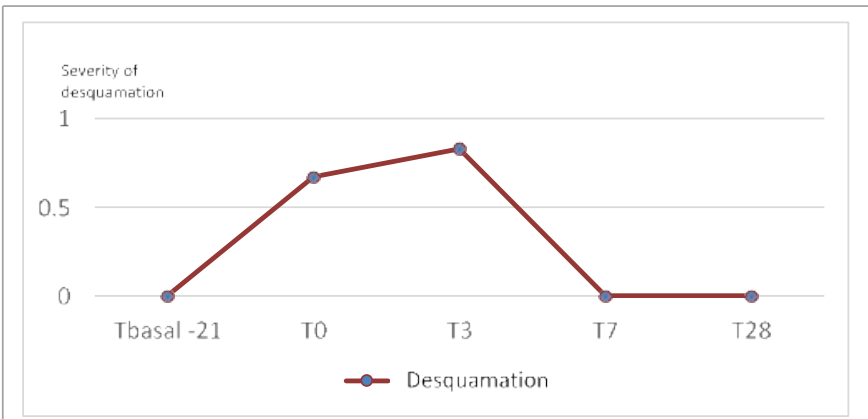

Figure 1: Evolution of desquamation (0-3) during the study.

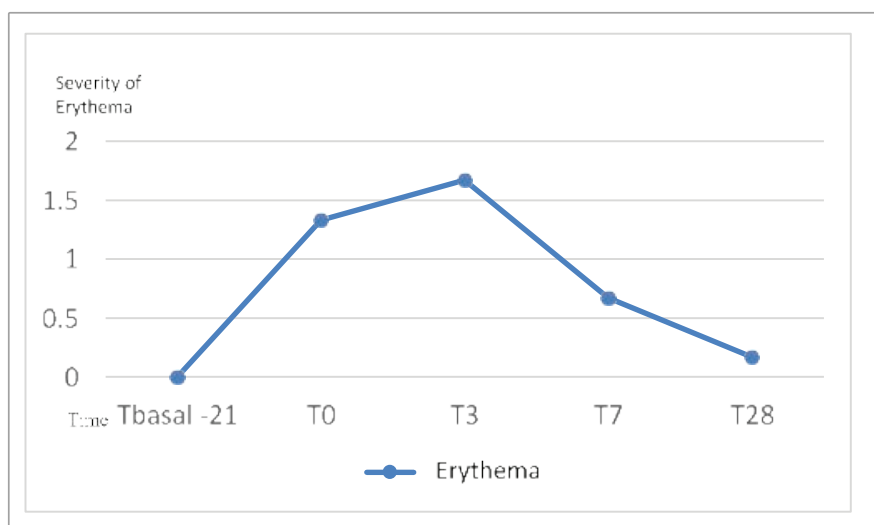

Figure 2: Evolution of erythema (0-3) during the study.

(Vitamin A), a precursor of retinoic acid [5]. Topical retinol is transformed into retinoic acid in a 2-step oxidation process and is less potent than pure retinoic acid (tretinoin) [6]. Topical retinol has shown remarkable anti-aging effects on three major types of skin cells: epidermal keratinocytes, dermal endothelial cells and fibroblasts [5, $2]$. Retinol is extremely unstable and easily degraded to biologically inactive forms upon exposure to light and air [6].

Kang S, et al. [7] showed that application of retinol on normal human skin induces epidermal thickening and enhances the expression of Cellular Retinoic Acid Binding Protein (CRABP II) and cellular retinoic binding protein (CRBP) mRNAs and proteins, as does retinoic acid [8].

One of the main adverse effects of retinoid treatment is irritant retinoid dermatitis. It can cause dermatitis, xerosis, dryness or peeling $[2,9]$. Kang S, et al. [7] also observed that retinol showed only minimal signs of erythema and irritation unlike tretinoin. In another study $(\mathrm{n}=6$; duration=14 days), Fluhr JW, et al. [10] confirmed that retinol produces considerably less Transepidermal Water Loss (TEWL), erythema and scaling than retinoic acid.

Although there is no exact conversion, it is accepted that retinol has one tenth the efficacy of pure retinoic acid [11]. We have previously published interesting antiaging results in wrinkles, firmness and viscoelasticity with excellent tolerance after one month use of a combination of high tolerance retinoids (retinol and Hydroxypinacolone Retinoate) and Deschampsia antarctica extract [12]. Interestingly, Duell EA, et al. [13] demonstrated that retinol could be as effective as retinoic acid in producing 'retinoid mediated histological changes' (like epidermal thickening and keratinocyte proliferation), but with much less irritancy.
Chemical peels consist in the application of a chemical substance on the skin with the aim of producing a controlled desquamation of the stratum corneum, epidermal renewal and a remodeling of the skin (neocollagenesis type I and IV) with subsequent improvement of skin appearance. It is one of the most frequently used procedures [14-16].

The term very superficial peels refers to those that only carry out a slight exfoliation of the stratum corneum and are usually called "Home Peel" [16].

Retinol, although considered a cosmetic active, when used at high concentrations can actually induce a peeling activity as shown by Sadick N, et al. [17]. We propose showed that a homecare use of high concentration retinol (1\%) under intensive use (three consecutive days, twice daily and double dose than in preparation phase) would induce a very superficial peel.

The adverse events of retinol peels include desquamation, erythema, burning sensation and/or tightness. They can also induce photosensitive reactions and a retinoid reaction. The 'retinoid reaction' has been found to be due to the free carboxylic acid in the polar end of the retinoid, thought to be initiated by release of proinflammatory cytokines such as IL-1, TNF- $\alpha$, IL-6, and IL-8 $[6,18]$.

\section{Aims}

The main purpose of this study was to assess the safety and tolerance of a new cosmetic protocol based on the use of a Home Peel with $1 \%$ retinol. The treatment will be considered to be well tolerated if TEWL is at normal levels (below $25 \mathrm{~g} / \mathrm{hm}^{2}$ ) within a week following the procedure and safe if patients do not report important adverse effects that require medical intervention. The secondary objective was to determine the efficacy of the protocol through improvement of clinical signs of photoaging according to subjective evaluations carried out by researcher and patients.

\section{Patients and Methods}

This was a prospective, case series, study. Six voluntary subjects were included in the study with aging between $35-55$ years (female) and facial skin aging (minimum assessment of 2 on the RAO-GOLDMAN scale: fine visible wrinkles).

\section{Treatment regimen}

The treatment protocol was divided into 3 stages. The duration of the study was 49 days. There were 5 visits (T-21 or T basal, T0, T3, T7 and T28), that were planned as shown in table 1.

Therefore, five products of the All Skin Med line made up the Home Peel protocol administered to the included patients. The products are considered safe and as a cosmetic product, their ingredients have an INCI nomenclature:

- The active formula All Skin Med Antiaging R Extreme Skin Renewal Gel [1.0], used for home peel contains $1 \%$ retinol as the main active ingredient. In addition, the formula has antioxidant ingredients (Vitamin $\mathrm{C}$ and $\mathrm{E}$ ), niacinamide, and an antipollution agent (Deschampsia antartica extract (Edafence ${ }^{\circledR}$ ).

- The transition formula (All Skin Med Antiaging R Skin Renewal Transition Cream) to prepare the skin for the home peel product, contains $0.02 \%$ retinol combined with antioxidants (Vitamin C and E), an anti-pollution agent (Edafence ${ }^{\circledast}$ ) and soothing agents (niacinamide and moisturizers).

- The product for skin hygiene, All Skin Med Purifying Gentle Cleansing Foam, contains deep seawater (rich in $\mathrm{Se}, \mathrm{Cu}$, 
Table 1: Study schedule.

\begin{tabular}{|c|c|c|c|c|}
\hline T basal (-21) & TO & T3 & T7 & T28 \\
\hline Recruitment basal visit & $\begin{array}{l}\text { Home peel with All } \\
\text { Skin Med Antiaging R } \\
\text { Extreme Skin Renewal } \\
\text { Gel [1.0], twice daily }\end{array}$ & $\begin{array}{l}\text { Home Peel procedure } \\
\text { ends }\end{array}$ & Post- procedure evaluation & End of the study \\
\hline Prepar & \multicolumn{2}{|l|}{ Home peel } & \multicolumn{2}{|l|}{ Post- procedure } \\
\hline $\begin{array}{l}\text { 1st week: All Skin Med Antiaging R } \\
\text { Extreme Skin Renewal Gel [1.0] } 2 \text { nights } \\
\text { a week and the rest of nights All Skin } \\
\text { Med Antiaging RTransition Cream } \\
\text { The following } 2 \text { weeks: All Skin Med } \\
\text { Antiaging R Extreme Skin Renewal Gel } \\
3 \text { nights a week and the rest of nights } \\
\text { All Skin Med Antiaging R Transition } \\
\text { Cream }\end{array}$ & $\begin{array}{l}\text { Cleansing: All Skin Med } \\
\text { Foam } \\
\text { Renovation: All Skin Med } \\
\text { Renewal Gel [1.0] day an } \\
\text { application) Regeneration } \\
\text { Rich Replenishing Cream } \\
\text { Photoprotection: During } \\
\text { Mineral Fluid SPF } 50\end{array}$ & $\begin{array}{l}\text { Purifying Gentle Cleansing } \\
\text { Antiaging R Extreme Skin } \\
\text { d night ( } 3 \text { to } 4 \text { pumps per } \\
\text { : All Skin Med Antiaging GF } \\
\text { day and night } \\
\text { the day All Skin Med }\end{array}$ & \multicolumn{2}{|c|}{$\begin{array}{l}\text { First } 10 \text { days: All Skin Med Antiaging R Transition } \\
\text { Cream at night and All Skin Med Antiaging GF Rich } \\
\text { Replenishing Cream in the morning. } \\
\text { Following days: All Skin Med Antiaging R Extreme } \\
\text { Skin Renewal Gel [1.0] at night, } 3 \text { nights per } \\
\text { week, alternating with All Skin Med Antiaging R } \\
\text { Transition Cream the rest of nights a week and All } \\
\text { Skin Med Antiaging GF Rich Replenishing Cream in } \\
\text { the morning. } \\
\text { Photoprotection: During the day All Skin Med } \\
\text { Mineral Fluid SPF } 50\end{array}$} \\
\hline
\end{tabular}

$\mathrm{Mn}, \mathrm{Ca}$ and $\mathrm{Zn}$ ) and plant extracts as purifying ingredients, hypoallergenic.

- All Skin Med Antiaging GF Rich Replenishing Cream is a regenerating cream rich in growth factors, with moisturizing and soothing activity.

- The All Skin Med Mineral Fluid SPF 50 antioxidant and photo protector used daily during the study contains Fernblock ${ }^{\bullet}$ and $100 \%$ mineral filters, providing complete spectrum protection against UVA, UVB, Visible light and Infrared (IR-A).

\section{Evaluations}

At all subject visits, clinical assessment, Reveal ${ }^{\oplus}$ photography and Transepidermal Water Loss (TEWL), were carried out. Tewameter ${ }^{\circ}$ TM300 (Courage \& Khazaka electronic) allow to determine the TEWL through the stratum corneum, as a measure of skin hydration and integrity of the skin barrier.

Severity of adverse effects Score: Desquamation (0-3), Erythema (0-3), Burning (0-3), Tightness (0-3), Others. 0: Null, 1: Mild, 2: Moderate, 3: Intense.

Severity of photoaging perceived by subject: Rated scores from 0 To 3. (None (0), Slight (1), Moderate (2) and Severe (3).

Severity of photoaging perceived by researcher: According to the validated RAO GOLDMAN (RGWS) 5-point scale: (1) without wrinkles, (2) visible but fine wrinkles, (3) moderately deep wrinkles, (4) deep wrinkles with defined edges and (5) very deep wrinkles with defined grooves. For its evaluation, Reveal photography was used.

Improvement perceived by researcher: IGA (-2 to 3$)$ : 0 : None; 1: minimal improvement; 2: moderate improvement; 3: intense improvement; -1: minimum worsening; -2: great worsening.

Improvement perceived by subjects: PGA ( -2 to 3$)$ : 0 : None; 1: minimal improvement; 2: moderate improvement; 3: intense improvement; -1: minimum worsening; -2: great worsening.

\section{Statistical study}

For efficacy variables of ordinal nature, such as clinical assessment of the degree of aging perceived by patient and researcher (RGWS), degree of improvement perceived by patient (PGA), degree of improvement perceived by researcher (IGA) and intensity of adverse effects, in each of the experimental times, a nonparametric Wilcoxon test was performed to see if there were significant differences. In addition, their frequencies and mean values were studied as reported.

\section{Results}

All six voluntary subjects signed the inform consent and finished the study.

Safety and tolerance of the protocol was confirmed. No significant increase in desquamation after finishing the 3-day peel, was obtained $(\mathrm{p}=0.65)$. In T7, and at the end of the treatment, none of the subjects presented any desquamation (all grade 0) (Figure 1).

\section{Erythema}

No significant increase in erythema was detected after the preparation phase nor after finishing the 3 - day peel (T3) ( $\mathrm{p}=0.41)$. In T7, the degree of erythema decreased being grade 1 in four subjects and the rest grade 0 . At the end of treatment (T28) only one subject presented grade of erythema 1 and the rest of patients grade of null erythema (0) (Figure 2).

\section{Burning}

After finishing the home- peel (T3), all had some degree of burning with an average of 1.33 , value lower with respect to T0 but without reaching significant difference $(\mathrm{p}=0.56)$. Burning sensation was therefore increase during the preparation phase but afterwards the adaptation let to a lesser degree of burning during the home peel phase. At the end of the study (T28) all the subjects obtained a degree of null burning. Although most of the subjects showed some degree of burning after the Home Peel treatment, without significant differences, all finished the treatment without burning, which shows the high tolerance to the treatment (Figure 3).

\section{Tightness}

After 21 days of preparation an average of 1.83 without reaching statistical significance $(\mathrm{p}=0.15)$, was observed. Although, majority of the subjects showed some degree of tightness after the Home Peel treatment, all of them finished the treatment without feeling of tightness, which shows the high tolerance to the treatment (Figure 4).

Next, the joint graph of all the registered evaluations on the different adverse effects, in the different temporal moments is shown. 


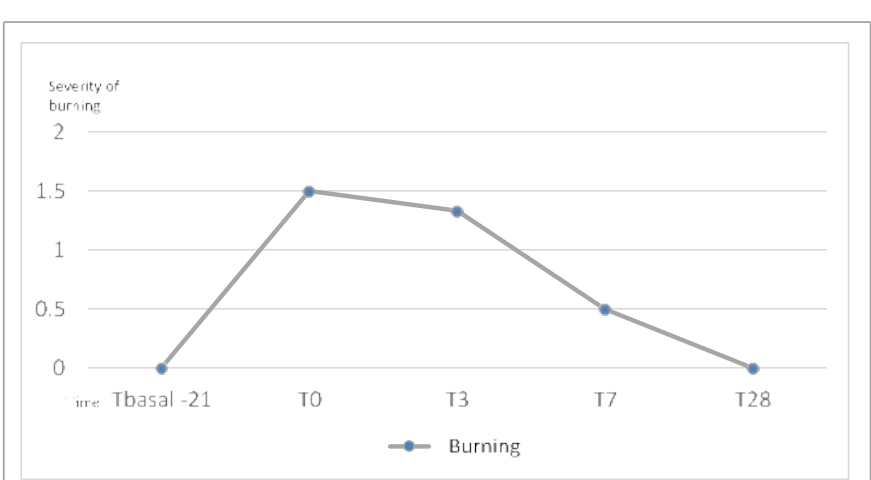

Figure 3: Evolution of burning sensation (0-3) during the study.

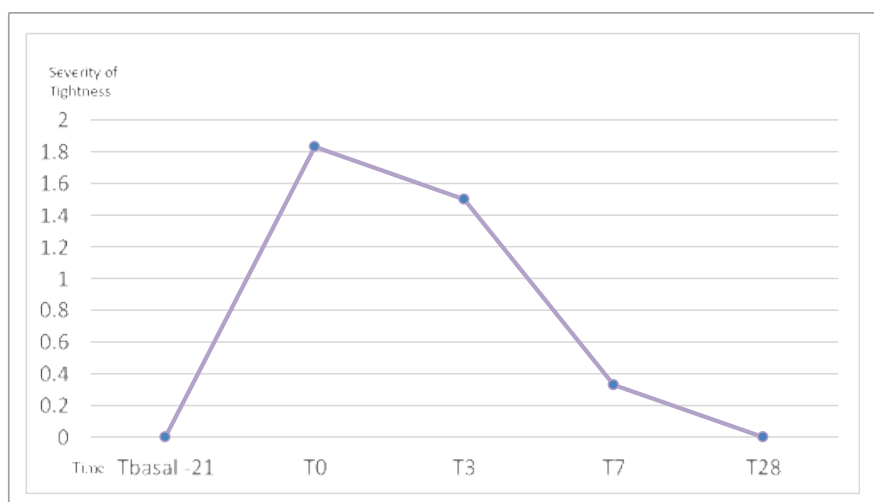

Figure 4: Evolution of tightness (0-3) during the study.

It is observed how the highest degree of tightness and burning was observed at the end of the preparation period (T0). While the highest degree of erythema and scaling was detected at the end of the homepeel (T3) and was progressively decreasing in the following visits of the study (Figure 5).

TEWL, as expected, varied throughout the study on the different areas. In the preparation period, non-significant change on different areas was detected. The highest increase in TEWL was observed after finishing the home-peel treatment period (T3) without significant difference between the evaluated areas $(\mathrm{p}=0.08,0.08$ and 0.22 , respectively in forehead, right and left cheek). But when compared T3 vs T0, a statistical increase in TEWL was reached in both cheeks between T3 vs T0 ( $\mathrm{p}=0.028$ in both of them). No statistical difference was reached in the forehead area $(\mathrm{p}=0.116)$.

At T7, the increment of TEWL compared to T0 was already nonsignificant in neither forehead nor the cheeks, suggesting a quick normalization of the TEWL.

At the end of the study (T28) the mean levels of TEWL were still higher compared to the initial evaluation: forehead 22.0, right cheek 18.6 and left cheek 16.1, but no significant differences were found ( $\mathrm{p}=0.08,0.53$ and 0.34 respectively) (Figure 6).

Regarding efficacy, the semi quantitative evaluations, the improvement perceived by the patient (PGA) with respect to aging was considered moderate (grade 2 ) in almost all patients and only one patient was considered mild (1). (Average 1.83). $83 \%$ of the patients obtained a moderate improvement after treatment. The improvement perceived by the researcher (IGA) was also considered moderate (grade 2) in all patients and mild (1) in only one patient. (Average

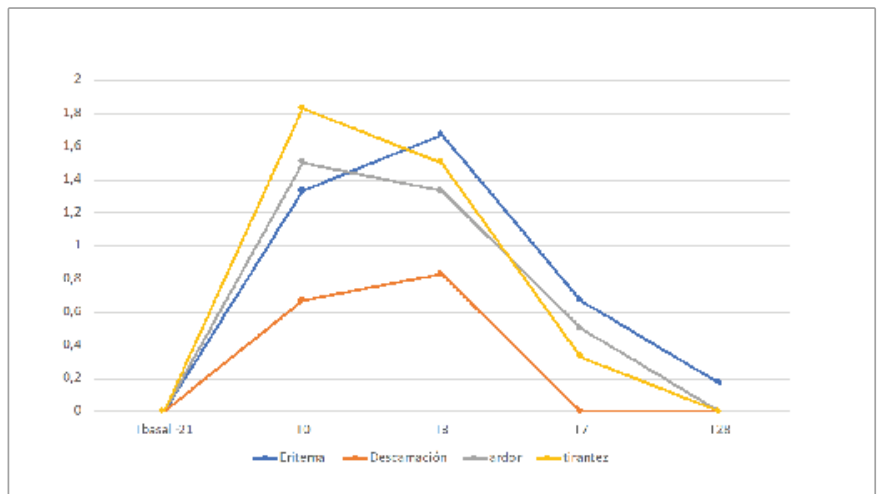

Figure 5: Evolution of erythema, desquamation, burning sensation and tightness (0-3) during the study.

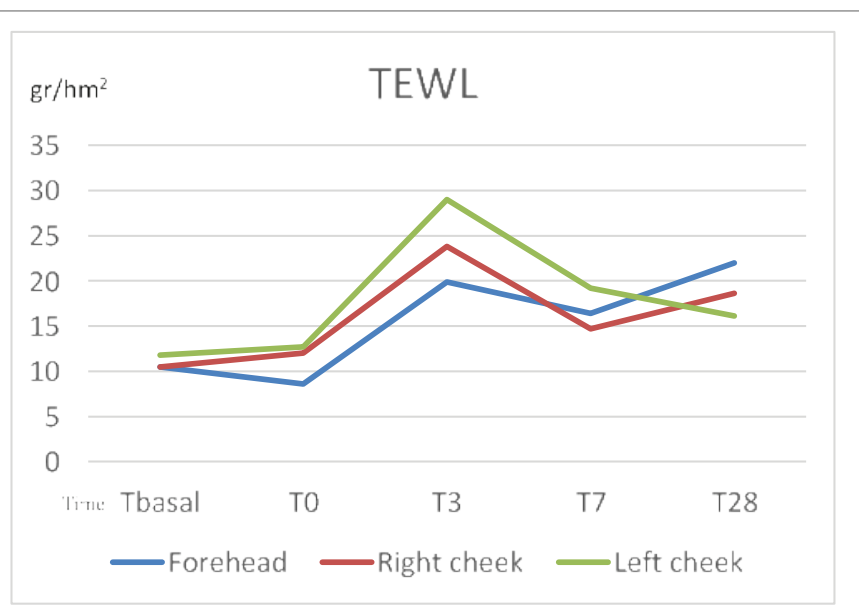

Figure 6: Evolution of TEWL $\left(\mathrm{gr} / \mathrm{hm}^{2}\right)$ during the study.

1.83). Non-significant differences in photoaging were found according to the RAO-Goldman scale. We attribute this to the low sensitivity of this scale to perceive slight changes and short study duration with few subjects treated (Figure 7).

\section{Discussion and Conclusion}

The use of retinoids has shown efficacy in the treatment of intrinsic and extrinsic aging [10].

The improvement at a cosmetic level induced by retinol is determined by the combined effect at different levels, at the level of dermal collagen stimulation (improving wrinkles), as well as the regulation of keratinocyte epidermal proliferation (improving roughness) and also the ability to inhibit the production of tyrosinase (improving pigmentation and dyschromiat) [11].

One of the main adverse effects of the use of classically- described retinoids is with respect to tolerance. It is therefore very interesting to note how by this protocol the tolerance was excellent, with adverse effects of less intensity than expected in peeling, erythema, burning and tightness.

After the preparation period, the greatest increase was seen in both TEWL and subjective adverse effects (tightness and burning sensation), but in no case the increase was statistically significant. Subsequently in the home-peeling phase itself (T0-T3), TEWL, desquamation 


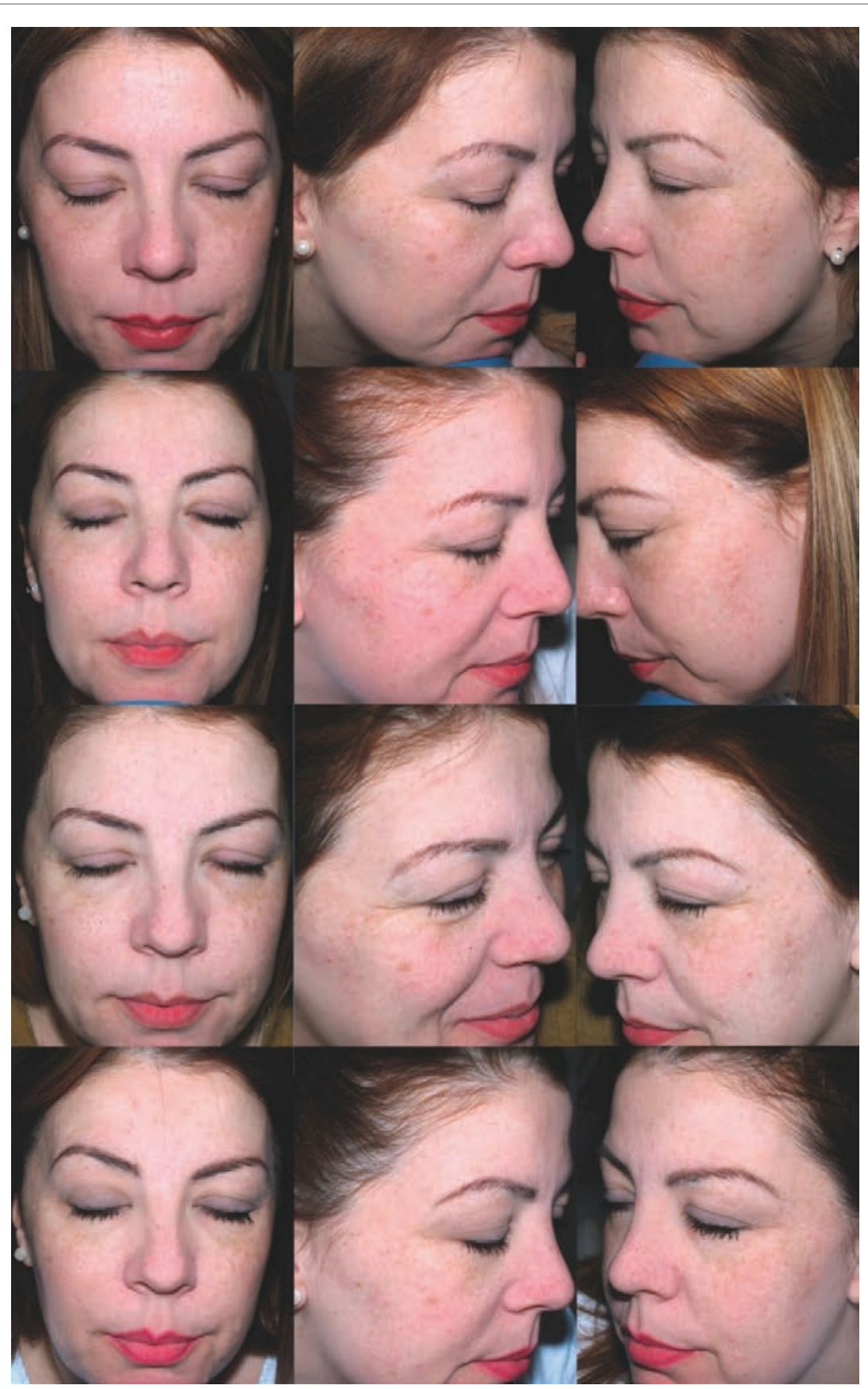

Figure 7: Patient number 2, after preparation phase (Tbasal) (upper pictures), right after the home peel (T3, middle pictures) and after treatment (T28) (lower pictures). Lighter spots and more luminosity were detected after treatment.

and erythema continued to increase but without reaching statistical significance. It is striking how already in the treatment period a decrease was observed in the most subjective sensations (burning and tightness), although no statistically significant differences were obtained here either. The preparation phase is fundamental to gain greater tolerance to the procedure.

As shown in T3, all side effects gradually decreased until they became null at the end of the study, except for slight erythema (mean 0.17). The TEWL did not return to basal levels but remained at slightly higher values, without statistical significance or clinical consequence. This supports that retinol treatment and even more with Home peel protocol, induces a greater TEWL and therefore is necessary to moisturize skin during treatment.

The objective changes of erythema and desquamation are hardly observed or they vary in a very light degree during the preparation time, but logically, they increase more or the end of home peels. In this case the intensification of treatment (twice a day) is in this case capable to produce a greater degree of erythema and desquamation. However, the increase in erythema and desquamation remains very mild and does not reach the moderate degree.

TEWL refers to the modification of the skin barrier by an extensive water loss. During the preparation phase (T0), although the patients 
described burning and tightness sensation, the TEWL did not increase significantly. It means that this preparation regime is objectively well tolerated as no significant changes were detected in TEWL, neither in erythema nor in desquamation. However, as happened with erythema and desquamation, the TEWL does present its largest increase after the home-peel (T3), reaching significant difference compared to T0 in both cheeks. This reflects the intensification in the retinol treatment regimen, which modifies the cutaneous barrier when a stricter regimen is used. At the end of the study, the values are slightly higher compared to $\mathrm{T}$ basal, evidencing that the structural changes in the cutaneous barrier are the ones that take longer to recover completely. However, this increment is not significant suggesting a good recovery of the skin conditions. In addition, the non- significant increment of TEWL at T7 $v s$. T0, suggests that already at T7 (only 4 days after finishing the home peel), the epidermal barrier is still in recovering.

In summary, the subjective measurements of burning and tightness are those that first begin to decline, followed by the variables of erythema and desquamation until practically null at T28. TEWL, because it is the most sensitive measurement, does not return to baseline measurements, although there are no statistically significant differences.

Regarding efficacy, it is concluded that the treatment is effective because the degree of improvement perceived by both patient and researcher was moderate in almost all patients.

In conclusion, the reported protocol for the management of skin aging seems to represent an effective cosmetic treatment because of the positive improvement in the signs of aging was perceived by the treated subjects and recovered by Investigator.

Finally it has to be underlined that, as shown, the preparation phase is essential to achieve a greater tolerance to the treatment with a subsequent positive follow-up. This excellent tolerance suggests that the reported protocol can be suitably used as an anti-aging protocol for home use.

\section{Conflicts of Interest}

This work was supported in part by Grants from Industrial Farmaceutica Cantabria. In addition, Dr Truchuelo is scientific advisor to Industrial Farmaceutica Cantabria.

\section{References}

1. Ruiz Martínez MA, Morales Hernández ME (2015) Aproximación al tratamiento del envejecimiento cutáneo. Ars Pharm 56: 183-191.

2. Darlenski R, Surber C, Flurhr JW (2010) Topical retinoids in the management of photodamaged skin: from theory to evidencebased practical approach. Br J Dermatol 163: 1157-1165.

3. Geria AN, Lawson CN, Halder RM (2011) Topical retinoids for pigmented skin. J Drugs Dermatol 10: 483-489.

4. Stefanaki C, Stratigos A, Katsambas A (2005) Topical retinoids in the treatment of photoaging. J Cosmet Dermatol 4: 130-134.

5. Shao Y, He T, Fisher GJ, Voorhees JJ, Quan T (2017) Molecular basis of retinol anti-aging properties in naturally aged human skin in vivo. Int J Cosmetic Sci 39: 56-65.
6. Mukherjee S, Date A, Patravale V, Korting HC, Roeder A, et al. (2006) Retinoids in the treatment of skin aging: an overview of clinical efficacy and safety. Clin Interv Aging 1: 327-348.

7. Kang S, Duell EA, Fisher GJ, Datta SC, Wang ZQ, et al. (1995) Application of retinol to human skin in vivo induces epidermal hyperplasia and cellular retinoid binding proteins characteristic of retinoic acid but without measurable retinoic acid levels or irritation. J Invest Dermatol 105: 549-556.

8. Bielli A, Scioli MG, D’Amico F, Tarquini C, Agostinelli S, et al. (2019) Cellular retinoic acid binding protein- II expression and its potential role in skin aging. Aging (Albany NY) 11: 1619-1632.

9. Griffiths CE, Kang S, Ellis CN, Kim KJ, Finkel LJ, et al. (1995) Two concentrations of topical tretinoin (retinoic acid) cause similar improvement of photoaging but different degrees of irritation. A double-blind, vehicle-controlled comparison of $0.1 \%$ and $0.025 \%$ tretinoin creams. Arch Dermatol 131: 1037-1044.

10. Fluhr JW, Vienne MP, Lauze C, Dupuy P, Gehring W, et al. (1999) Tolerance profile of retinol, retinaldehyde and retinoic acid under maximized and long-term clinical conditions. Dermatology 199: 5760.

11. Riahi R, Bush AE, Cohen PR (2016) Topical Retinoids: Therapeutic Mechanisms in the Treatment of Photodamaged Skin. Am J Clin Dermatol 17: 265-276.

12. Perez Davó A, Truchuelo MT, Vitale M, Gonzalez-Castro J (2019) Efficacy of an Antiaging Treatment Against Environmental Factors: Deschampsia antarctica Extract and High-tolerance Retinoids Combination. J Clin Aesthet Dermatol 12: E65- E70.

13. Duell EA, Derguini F, Kang S, Elder JT, Voorhees JJ (1996) Extraction of human epidermis treated with retinol yields retro-retinoids in addition to free retinol and retinyl esters. J Invest Dermatol 107: 178-182.

14. Truchuelo M, Cerda P, Fernandez LF (2017) Chemical Peeling: A Useful Tool in the Office. Actas Dermo-Sifiliográficas 108: 315-322.

15. Okano Y, Abe Y, Masaki H, Santhanam U, Ichihashi M, et al. (2003) Biological effects of glycolic acid on dermal matrix metabolism mediated by dermal fibroblasts and epidermal keratinocytes. Exp Dermatol 12: 57-63.

16. Stegman SJ (1982) A comparative histologic study of the effects of three peeling agents and dermabrasion on normal and sundamaged skin. Aesthetic Plast Surg 6: 123-135.

17. Sadick N, Edison BL, John G, Bohnert KL, Green B (2019) An Advanced, Physician-Strength Retinol Peel Improves Signs of Aging and Acne Across a Range of Skin Types Including Melasma and Skin of Color. J Drugs Dermatol 18: 918-923.

18. Kong R, Cui Y, Fisher GJ, Wang X, Chen Y, et al. (2016) Comparative study of the effects of retinol and retinoic acid on histological, molecular, and clinical properties of human skin. J Cosmet Dermatol 15: 49-57. 